Revue pluridisciplinaire d'études médiévales

\title{
Aux marges des manuscrits. Éléments pour une étude de la réception des Grandes Chroniques de
} France

\section{Antoine Brix}

\section{(2) OpenEdition Journals}

Édition électronique

URL : http://journals.openedition.org/questes/4419

DOI : $10.4000 /$ questes.4419

ISSN : 2109-9472

Éditeur

Les Amis de Questes

\section{Édition imprimée}

Date de publication : 20 juin 2017

Pagination : 59-83

ISSN : 2102-7188

\section{Référence électronique}

Antoine Brix, «Aux marges des manuscrits. Éléments pour une étude de la réception des Grandes Chroniques de France », Questes [En ligne], 36 | 2017, mis en ligne le 02 juillet 2017, consulté le 24 mars 2020. URL : http://journals.openedition.org/questes/4419; DOI : https://doi.org/10.4000/ questes. 4419 


\title{
Aux marges des manuscrits. Éléments pour une étude de la réception des Grandes Chroniques de France
}

\author{
Antoine BRIX \\ Université catholique de Louvain - F.R.S.-FNRS
}

Les travaux de Bernard Guenée sont sans conteste fondateurs dans le domaine des études sur la diffusion et la réception des ouvrages littéraires médiévaux, lesquelles se sont multipliées depuis la fin du $\mathrm{XX}^{\mathrm{e}}$ siècle ${ }^{1}$. Dans un article qu'il consacre à la fortune de cette œuvre à succès $^{2}$ que furent les Faits des Romains, Bernard Guenée expose à grands traits ce qui deviendra un champ de recherche à part entière :

\footnotetext{
${ }^{1}$ Différents genres littéraires ont été abordés. Par exemple, et chronologiquement : Pierre-Yves Badel, Le Roman de la Rose au XIV ${ }^{e}$ siècle. Étude de la réception de l'œuvre, Genève, Droz, 1980 ; Matthias M. Tischler, Einharts Vita Karoli. Studien zur Entstehung, Überlieferung und Rezeption, Hannovre, Hahn, 2001, 2 vol.; Christopher Allmand, The De Re militari of Vegetius. The reception, transmission and legacy of a Roman text in the Middle Ages, Cambridge, Cambridge University Press, 2011 ; Noëlle-Laetitia Perret, Les Traductions françaises $d u$ De Regimine principum de Gilles de Rome. Parcours matériel, culturel et intellectuel d'un discours sur l'éducation, Leiden/Boston, Brill, 2011 ; Godfrey of Viterbo and his readers. Imperial tradition and universal history in late medieval Europe, dir. Thomas Foerster, Farnham, Ashgate, 2015 ; Christine Gadrat-Ouerfelli, Lire Marco Polo au Moyen Âge. Traduction, diffusion et réception du Devisement du monde, Turnhout, Brepols, 2015.

2 Comme d'autres, je préfère au terme de best-seller, parfois employé en médiévistique, celui, moins connoté, d' « œuvre à succès ». Best-seller, participant des champs lexicaux de l'économie de marché, du commerce et des classements, est un vocable qui rend compte de façon assez erronée des voies du succès littéraire médiéval, qui est inscrit dans le temps long et ne dépend pas des possibilités de reproductibilité à grande échelle. Les différentes études entreprises dans le cadre du projet de recherche OPUS («Euvres pieuses vernaculaires à succès »), mené à l'échelle européenne entre 2010 et 2015, ont envisagé plusieurs modalités du succès littéraire au Moyen Âge.
} 
Dans ses derniers développements, l'histoire de l'historiographie médiévale a pris conscience qu'il ne suffisait pas d'étudier la composition d'une œuvre historique et de situer celle-ci par rapport à son auteur, mais qu'il fallait aussi en définir le public ${ }^{3}$.

Les médiévistes sont invités de la sorte à dépasser une conception plus traditionnelle qu'a notamment formulée, une quarantaine d'années auparavant, le chartiste Robert Bossuat : «L'historiographie n'est, à la vérité, qu'une branche de l'histoire littéraire ${ }^{4} »$. Se dessine ainsi une perspective stimulante pour les spécialistes de l'écriture historique médiévale: secouer le joug des considérations strictement littéraires de composition et d'intention, et ouvrir les recherches vers des horizons neufs d'histoire culturelle et sociale.

Mais derrière la volonté, énoncée très simplement par Bernard Guenée, de «définir le public» des ouvrages historiographiques médiévaux, c'est en réalité toute la complexité de l'étude du devenir des œuvres littéraires dans le temps qui se trouve dissimulée; les problèmes heuristiques, méthodologiques et interprétatifs abondent. La définition du public peut en effet être envisagée à deux niveaux différents au moins, chacun mettant en œuvre des perspectives multiples: la diffusion et la réception. Il est à mon sens utile de distinguer ces deux termes, diffusion et réception ${ }^{5}$, que l'on a coutume

${ }^{3}$ Bernard Guenée, «La culture historique des nobles. Le succès des Faits des Romains (XIII ${ }^{\mathrm{e}}-\mathrm{XV}^{\mathrm{e}}$ siècles) », dans La Noblesse au Moyen Âge. XI ${ }^{e}-X V^{e}$ siècles. Essais à la mémoire de Robert Boutruche, dir. Philippe Contamine, Paris, PUF, 1976, p. 261-288, cit. p. 261.

${ }^{4}$ Robert Bossuat, «Leçon d'ouverture du cours de sources narratives et littéraires de l'histoire de France à l'École des chartes (3 novembre 1937) », Bibliothèque de l'École des chartes, vol. 99, 1938, p. 132-150, cit. p. 137.

5 Les travaux de l' «école de Constance » relatifs à la réception des ouvrages littéraires, dus en particulier à Hans Robert Jauss et Wolfgang Iser, ont suscité des débats sur lesquels il n'y a pas lieu de revenir ici. Pour une perspective critique - 
d'employer l'un pour l'autre de façon indistincte, de juxtaposer dans un binôme stéréotypé et ambigu, et d'intervertir à l'envi. Chercher à lever l'équivoque lexicale brouillant les pistes de la diffusion et de la réception constitue un préalable nécessaire à la bonne compréhension du titre donné au présent article.

\section{Diffusion, réception, lectorat}

\section{Diffusion et réception}

La définition de la diffusion dans le contexte des études médiévales est instinctive et un emprunt aux dictionnaires du français courant suffit à en formuler une : «[En parlant d'un ouvrage imprimé, d'un journal, d'un tract, etc., et $p$. ext., d'un disque ou d'un film] Action de le distribuer dans le public ${ }^{6} »$. Étudier la diffusion d'une œuvre médiévale, c'est dès lors s'intéresser aux réseaux et canaux de circulation des manuscrits considérés comme artefacts et comme produits manufacturés, aux acteurs du monde des manuscrits en ce qu'ils les commandent, les copient, les achètent, les possèdent et les abritent dans leurs bibliothèques. Ainsi conçue comme un ensemble de phénomènes matériels, la diffusion n'implique, dans le chef du «public », aucune activité intellectuelle directement liée aux contenus transmis, ni aucune familiarité avec eux.

Se limiter strictement à des données relatives à la diffusion ne permet pas toujours de «définir le public» d'une façon qui soit satisfaisante, en ce qu'un dénombrement s'accompagne idéalement de

quoique ancienne-, voir La Réception de l'œuvre littéraire. Recueil d'études du colloque organisé par l'université de Wrocław, dir. Józef Heistein, Wrocław, Wydawnictwo Uniwersytetu Wrocławskiego, 1983.

6 ATILF - CNRS \& Université de Lorraine, Le Trésor de la langue française informatisé, art. «diffusion », 2004, URL: http://atilf.atilf.fr, page consultée le 12 mai 2017. 
quelque caractérisation des individus ainsi dénombrés. Certes, cette approche du public par le strict biais de la diffusion est capitale et sert l'histoire des bibliothèques médiévales ; cependant, elle n'épuise pas la définition du public, qui gagne à prendre en considération, outre la diffusion, le volet de la réception à proprement parler.

La réception, dans l'acception que je lui préfère, est un phénomène à la fois intellectuel et matériel qui résulte de la pratique effective du livre comme contenu ; ce sont les idées et les pratiques qui, chez le ou les sujets lisant un texte, procèdent de cet acte de lecture. On peut rapprocher la réception de ce que la linguistique ou la théorie de la communication désignent de ce terme même : l'action de recevoir un message et le résultat de cette action.

La réception n'est pas directement perceptible aux yeux de l'historien. Liée à la consultation des manuscrits et à leur lecture, elle ne laisse, le plus souvent, aucune trace. Cependant, certaines pratiques des lecteurs médiévaux laissent parfois entrevoir la réception d'une œuvre, qui à l'occasion se manifeste matériellement par des annotations. Laissées dans les manuscrits de l'œuvre elle-même ou se rapportant au contenu de celle-ci dans les marges d'autres manuscrits, ces annotations constituent la matière première des études de réception. Une acception large des indices de la réception d'une œuvre inclut en outre le commentaire séparé et la traduction, voire - bien qu'il soit difficile d'établir des filiations certaines - la réélaboration et la réécriture des contenus transmis au sein d'une compilation nouvelle ou la mise en œuvre de ceux-ci dans le cadre d'une activité professionnelle ou d'un loisir. 


\section{Lecteurs et manuscrits des Grandes Chroniques}

On connaît assez bien la diffusion des manuscrits des Grandes Chroniques de France ${ }^{7}$, qui avec 130 témoins subsistants constituent l'une des œuvres les plus largement conservées du Moyen Âge français $^{8}$. La moitié de ce volumineux corpus est enluminée ${ }^{9}$. La fraction enluminée de la tradition manuscrite est pour une large partie constituée de manuscrits luxueux qui ont attiré l'attention des historiens de l'art comme des historiens. Cet intérêt a permis d'identifier les possesseurs d'un grand nombre de manuscrits enluminés des Grandes Chroniques et, en leur adjoignant les mentions d'exemplaires perdus connues par le dépouillement des inventaires anciens, de dresser un panorama relativement satisfaisant de leur diffusion. Il n'est pas surprenant, au regard des manuscrits considérés, que l'aristocratie bibliophile et les princes de sang royal, à qui la lecture des Grandes Chroniques avait été plusieurs fois recommandée au cours des $\mathrm{XIV}^{\mathrm{e}}$ et $\mathrm{XV}^{\mathrm{e}}$ siècles $^{10}$, se taillent dans cet aperçu la part du lion.

\footnotetext{
${ }^{7}$ Les Grandes Chroniques de France, éd. Jules Viard, Paris, Société de l'histoire de France, 1920-1953, 10 vol. ; Les Grandes Chroniques de France. Chronique des règnes de Jean II et de Charles $V$, éd. Roland Delachenal, Paris, Société de l'histoire de France, 1910-1920, 4 vol. Sur la genèse des Grandes Chroniques, voir notamment Gabrielle M. Spiegel, The Chronicle Tradition of Saint-Denis. A survey, Brookline/Leyden, Classical folia editions, 1978 ; et, en dernier lieu, Bernard Guenée, Comment on écrit l'histoire au XIII siècle. Primat et le Roman des roys, éd. Jean-Marie Moeglin, Paris, CNRS, 2016. Sur les continuations données au Roman de Primat par l'abbaye de Saint-Denis, voir Isabelle Guyot-Bachy et JeanMarie Moeglin, «Comment ont été continuées les Grandes Chroniques de France dans la première moitié du $\mathrm{XIV}^{\mathrm{e}}$ siècle », Bibliothèque de l'École des chartes, vol. $163 / 2,2005$, p. 385-433.

${ }^{8}$ Lectures françaises de la fin du Moyen Âge. Petite anthologie commentée de succès littéraires, éd. Frédéric Duval, Genève, Droz, 2007.

9 À propos des manuscrits enluminés des Grandes Chroniques, voir Anne D. Hedeman, The Royal Image. Illustrations of the "Grandes Chroniques de France”. 1274-1422, Berkeley/Los Angeles/Oxford, University of California Press, 1991.

${ }^{10}$ Jean Daudin en recommande la lecture à Charles $\mathrm{V}$ dans le prologue de sa traduction du De Remediis de Pétrarque (Ludmilla Evdokimova, "Charles V dans le miroir de deux traductions de Jean Daudin », dans Traduire au XIV siècle. Evrart
} 
Pourtant, caractériser la réception des Grandes Chroniques auprès de ce public en particulier confine à l'impossible. Leurs beaux manuscrits, au parchemin éclatant, sont muets quant à la réception, si bien que leur usage réel par les médiévaux a pu être mis en doute ${ }^{11}$. C'est que ce public est composé de « lecteurs amateurs », qui gagnent à être distingués - sans exclusivité toutefois - des «lecteurs professionnels $^{12} \gg$.

La séance de lecture publique, qui n'a pas disparu du contexte aristocratique à la fin du Moyen $\hat{A} g e^{13}$, caractérise le rapport du lecteur amateur aux textes et aux manuscrits. Pour ce personnage, le manuscrit est avant tout parole ; il est lu à haute voix dans un intérieur agréable -

de Conty et la vie intellectuelle à la cour de Charles $V$, dir. Joëlle Ducos et Michèle Goyens, Paris, Champion, 2015, p. 245-265, cit. p. 257 et 264), et Jean Gerson au futur Charles VII dans une lettre adressée à son précepteur (Jean Gerson, «Lettre 42 », dans Id., Euvres complètes, éd. Palémon Glorieux, Paris/Tournai/Rome/New York, Desclée, 1960, t. 2, p. 203-215, cit. p. 213, cité dans Jacques Krynen, L'Empire du roi. Idées et croyances politiques en France. XIII $-X V^{e}$ siècle, Paris, Gallimard, 1993, p. 215.

11 «Bien des manuscrits semblent n'avoir jamais été lus. Les manuscrits les plus luxueux, les manuscrits d'apparat sont avant tout la manifestation d'un statut social. Ils sont copiés pour être vus plus que pour être lus » (Lectures françaises de la fin du Moyen Âge, op. cit., p. 19). L'usure et la saleté des feuillets d'un codex doivent être considérés comme des indices d'utilisation et de réception, ainsi que l'a montré l'étude de Kathryn M. Rudy, «Dirty books. Quantifying patterns of use in medieval manuscripts using a densitometer», Journal of historians of Netherlandish art, vol.2/1-2, 2010, URL: http://www.jhna.org/index.php/pastissues/volume-2-issue-1-2/129-dirty-books, page consultée le 12 mai 2017.

${ }^{12}$ Je reprends dans cette partie la distinction entre «lecteur amateur » et «lecteur professionnel » proposée par Céline Van Hoorebeeck, « Du livre au lire. Lectures et lecteurs à l'épreuve des catégorisations sociales », dans Lecteurs, lectures et groupes sociaux au Moyen Âge. Actes de la journée d'étude organisée par le Centre de recherche "Pratiques médiévales de l'écrit" (PraME) de l'Université de Namur et le département des manuscrits de la Bibliothèque royale de Belgique. Bruxelles, 18 mars 2010, dir. Xavier Hermand, Étienne Renard et Céline Van Hoorebeeck, Turnhout, Brepols, 2014, p. 123-131; Livres et lectures des fonctionnaires des ducs de Bourgogne (ca. 1420-1520), Turnhout, Brepols, 2014, p. 303-309.

${ }^{13}$ Voir Jacques Krynen, L'Empire du roi, op. cit., p. 312-313, et plus généralement Joyce Coleman, Public Reading and the reading public in late medieval England and France, Cambridge, Cambridge University Press, 1996. 
chambre, grande salle, bibliothèque ${ }^{14}$. La Chronique du bon duc Loys de Bourbon témoigne de ce type de séances, au cours desquelles les Grandes Chroniques - ou un ouvrage proche ${ }^{15}$ - étaient lues à haute voix pour un public plus ou moins nombreux. En effet, elle relate que Louis II de Bourbon, beau-frère de Charles V et oncle de Charles VI,

pour avoir plus haulte mémoire, faisoit lire à son disner continuellement les gestes des trèsrenommés princes, jadis rois de France, et d'aultres dignes d'honneur, et en ce se délectoit après le service divin, duquel l'office il disoit très révéremment ${ }^{16}$.

${ }^{14}$ À propos de la lecture en milieu aristocratique, voir Hélène Haug, Fonctions et pratiques de la lecture à la fin du Moyen Âge. Approche sociolittéraire du discours sur la lecture en milieu curial d'après les sources narratives françaises et bourguignonnes (1360-1480), Thèse de doctorat, Louvain-la-Neuve, Université catholique de Louvain, 2013.

${ }^{15}$ Les mentions de « chroniques », « gestes » ou « histoires de France » - et autres énoncés similaires -, abondantes dans les sources littéraires et documentaires du bas Moyen Âge, ne peuvent pas être considérées comme faisant unanimement référence aux Grandes Chroniques sans de sérieuses précautions critiques. Par exemple, faut-il croire qu'il songe aux Grandes Chroniques lorsque Jean Wauquelin renvoie le lecteur de son Girart de Roussillon aux «vrayes croniques de France » ou aux «histoires des rois de France » (Jean Wauquelin, Cronicques des faiz de feurent monseignr Girart de Rossillon, éd. Léonce de Montille, Paris, Champion, 1880, p. 28 et 361) ? C'est l'hypothèse d'Elizabeth J. Moodey, Illuminated Crusader Histories for Philip the Good of Burgundy, Turnhout, Brepols, 2012, p. 64. À l'inverse, les Croniques de France que Marguerite de Bécourt lègue dans son testament, en 1460, à son cousin Jean de Rubempré, seraient plutôt un exemplaire de la Chronique abrégée de Guillaume de Nangis (Bertrand Schnerb, «Les livres de Marguerite de Bécourt, dame de Santes », dans Livres et lectures de femmes en Europe entre Moyen Âge et Renaissance, dir. AnneMarie Legaré, Turnhout, Brepols, 2007, p. 211-220, cit. p. 213 et 220, n. 18).

${ }^{16}$ La Chronique du bon duc Loys de Bourbon, éd. Martial-Alphonse Chazaud, Paris, Renouard, 1876, p. 273 ; cité dans Françoise Autrand, Jean de Berry. L'art et le pouvoir, Paris, Fayard, 2000, p. 311. Contrairement à certaines enluminures évoquant, par une mise en abyme, la réception de l'ouvrage qu'elles illustrent (notamment dans le Roman de la rose, comme le montre Lori Walters, "Reading the Rose. Literacy and the presentation of the Roman de la rose in medieval manuscripts », Romanic review, vol. 85/1, 1994, p. 1-26), celles conservées dans les manuscrits des Grandes Chroniques ne représentent pas de séances de lecture publique. L'une des quatre enluminures du frontispice du manuscrit Paris, Arsenal, ms. 5223, fol. 2r constitue néanmoins une exception. Elle représente une lecture en groupe, confinée à un environnement monastique - le lecteur comme les auditeurs portent la tonsure. Anne D. Hedeman, The Royal Image, op. cit., p. 233, décrit 
La très grande rareté des indices relatifs à la réception des Grandes Chroniques dans des manuscrits employés pour la lecture publique ne doit pas surprendre ${ }^{17}$. Les livres lus à haute voix n'ont pas vraiment l'occasion d'être annotés. «Dans ce modèle de lecturespectacle [...], les remarques et autres interventions générées par le récit $[\ldots]$ ont plutôt dû être formulées à voix haute et "en direct" par les auditeurs $^{18} \gg$.

L'un des moyens dont disposent les historiens pour déceler des traces matérielles de la réception des Grandes Chroniques de France est de se tourner vers les manuscrits d'aspect et d'origine plus modestes, ayant circulé dans des milieux différents de la grande aristocratie bibliophile. La diffusion des Grandes Chroniques a été si large qu'elle a dépassé les frontières de la noblesse pour devenir, à côté de ce que Bernard Guenée appelle le «fonds commun de la culture historique $^{19} »$, un classique des bibliothèques françaises. Ainsi, les contingences et déterminismes sociaux, qui limitent sociologiquement la diffusion de nombreux ouvrages, n'ont pas œuvré si pesamment contre celle des Grandes Chroniques. Bien des manuscrits sont parvenus dans des mains de « lecteurs professionnels ${ }^{20} »$.

Les caractéristiques des lecteurs professionnels rejoignent sur plusieurs points celles des «gens de savoir» étudiés par Jacques Verger $^{21}$. Ainsi lisent-ils non pas tant pour leur divertissement, leur formation ou leur culture que dans le cadre de leur fonction et dans

quant à elle cette enluminure comme mettant en scène un moine écrivant devant d'autres moines en discussion.

${ }^{17}$ Ce constat d'impuissance relative de l'historien est dressé par Bernard Guenée, Histoire et culture historique dans l'Occident médiéval, Paris, Aubier, 2011 [1980], p. 256.

${ }^{18}$ Céline Van Hoorebeeck, « Du livre au lire», art. cit., p. 130.

${ }^{19}$ Bernard Guenée, Histoire et culture historique, op. cit., p. 301-307.

${ }^{20}$ Céline Van Hoorebeeck, «Du livre au lire», art. cit.; Livres et lectures, op. cit.

${ }^{21}$ Jacques Verger, Les Gens de savoir dans l'Europe de la fin du Moyen Âge, Paris, PUF, 1997. 
l'exercice de leur profession. Souvent officiers de l'État, ils se rencontrent fréquemment au Parlement de Paris, à la Chambre des comptes ou à la chancellerie ${ }^{22}$. À la fin du Moyen Âge, ces institutions constituent de véritables viviers de lecteurs professionnels étudiant et annotant des manuscrits plus modestes dans le cadre très personnel de leur étude, de leur bureau ou de la petite bibliothèque qu'ils se sont constituée $^{23}$. Les Grandes Chroniques ont pénétré ces lieux d'études où leur contenu a pu servir des intérêts très différents.

Je présenterai ci-dessous trois cas de ce phénomène, documentés par les notes marginales que les utilisateurs ont laissées dans leurs manuscrits, témoins de «leurs soucis et leurs obsessions ${ }^{24} »$.

\section{Les réceptions des Grandes Chroniques}

\section{Insertion géographique spécifique}

Si les Grandes Chroniques se présentent elles-mêmes sous les traits d'une histoire française, narrée du point de vue francilien ${ }^{25}$, elles ont néanmoins joui d'une diffusion géographique variée. Certes,

\footnotetext{
${ }^{22}$ De nombreuses études monographiques ont été consacrées à ce personnel dans la perspective du développement d'un humanisme français aux XIV et $\mathrm{XV}^{\mathrm{e}}$ siècles. Voir, par exemple, le panorama brossé par Nicole Pons, «Les chancelleries parisiennes sous les règnes de Charles VI et Charles VII », dans Cancelleria $e$ cultura nel Medio Evo. Communicazioni presentate nelle giornate di studio della commissione, Stoccarda, 29-30 agosto 1985, XVI Congresso internazionale di scienze storiche, dir. Germano Gualdo, Vatican, Archivio segreto Vaticano, 1990, p. 137-168.

${ }^{23}$ Dans un passage éloquent d'une longue lettre à Gontier Col, le secrétaire du roi Jean de Montreuil se met en scène en son étude, le calamus à la main. Voir son Epistola 161, 1. 150-161 (Jean de Montreuil, Opera, éd. Ezio Ornato et al., Turin, Giappichelli, 1963-1975, 2 vol., cit. t. 1, p. 231-232); une traduction du passage concerné est donnée par Alfred Coville, Gontier et Pierre Col et l'humanisme en France au temps de Charles VI, Paris, Droz, 1934, p. 131, et Michel Mollat, Genèse médiévale de la France moderne. XIV $-X V^{e}$ siècle, Paris, Arthaud, 1977, p. 161.

${ }^{24}$ Bernard Guenée, Histoire et culture historique, op. cit., p. 237.

${ }^{25}$ Voir le prologue de Primat : Les Grandes Chroniques de France, éd. Jules Viard, op. cit., t. 1, p. 1-6.
} 
comme l'a souvent rappelé Bernard Guenée, le cours de la Loire n'a que rarement été franchi par les manuscrits des Grandes Chroniques ${ }^{26}$, mais aucune barrière n'a pu être mise en évidence aux marges septentrionales du royaume. En effet, les manuscrits des Grandes Chroniques ont été diffusés dans les Pays-Bas méridionaux et des indices de réception peuvent être relevés dans plusieurs exemplaires conservés, dont les manuscrits Bruxelles, KBR, 14910-14912, Paris, BnF, fr. 4937-4938 et nouv. acq. fr. 6225.

Le manuscrit Paris, BnF, nouv. acq. fr. $6225^{27}$ transmet le récit des Grandes Chroniques pour ce qui concerne le XIV siècle, depuis l'avènement de Philippe IV le Bel jusqu'à la mort de Charles V. Les rois concernés, derniers Capétiens directs et premiers Valois, ont été très actifs dans le nord de la France et dans ce qui a été, au XV $\mathrm{XV}^{\mathrm{e}}$ siècle, la moitié nord du «royaume inachevé » des Valois-Bourgogne ${ }^{28}$. D'ailleurs, notre manuscrit est fait d'un papier dont le filigrane, une tête de cerf vue de profil, rappelle des papiers produits dans la deuxième

\footnotetext{
${ }^{26}$ Voir Bernard Guenée, «Histoire d'un succès », dans Les Grandes Chroniques de France. Reproduction intégrale en fac-similé des miniatures de Fouquet. Manuscrit français 6465 de la Bibliothèque nationale de Paris, dir. François Avril, Marie-Thérèse Gousset et Bernard Guenée, [Paris], P. Lebaud, 1987, p. 81-138.

27 Papier; XV ${ }^{\mathrm{e}}$ s. ; $288 \times 202 \mathrm{~mm}$; 424 feuillets (36 sénions); 1 col. ; 291. Ce manuscrit a appartenu à Joseph Barrois qui l'a vendu au comte d'Ashburnham avec le reste de sa collection en 1849. Il ne figurait pas au nombre des 66 codices de Barrois que Léopold Delisle avait identifiés en 1883 comme ayant été volés à des dépôts français. En 1888, le libraire strasbourgeois Karl J. Trübner acquit cette partie de la collection Barrois et trouva avec la France un accord sur la restitution des manuscrits volés. Il acheta en outre, pour en faire don à la Bibliothèque nationale, le codex $n^{\circ} 373^{*}$ de la collection Barrois, qui est aujourd'hui conservé sous la cote Paris, BnF, nouv. acq. fr. 6225. Pourtant, ce manuscrit apparaît encore dans le catalogue de la vente des manuscrits Barrois du comte d'Ashburnham, prisés chez Sotheby's en juin 1901 (Catalogue of the portion of the famous collection of manuscripts, the property of the Rt. Hon. the Earl of Ashburnham, known as the Barrois collection which will be sold by auction by Messrs. Sotheby, Wilkinson \& Hodge [...] on Monday, the $10^{\text {th }}$ day of June, 1901, and four following days, Londres, J. Davy and sons, 1901, p. 50 [n 128]).

28 Élodie Lecuppre-Desjardins, Le Royaume inachevé des ducs de Bourgogne. $X I V^{e}-X V^{e}$ siècles, Paris, Belin, 2016.
} 
décennie $d u X v^{e}$ siècle à Malines, petite seigneurie enclavée dans le duché de Brabant ${ }^{29}$. Le texte est en outre rédigé dans une scripta présentant indéniablement des traits picards ${ }^{30}$ (par exemple, fol. $1 \mathrm{r}:$ : le premier capitle », «la ducee d'Acquitaine »; fol. 82r : «il approca »; fol. 94r : «grans monchiaux »; fol. 217r : «roy d'Engletiere »; fol. 422v : «la dicte ordenanche »). Un seing sur le dernier feuillet, «J. Hellevin », qui est celui d'un possesseur plutôt que d'un scribe, met ce codex en relation avec le manuscrit Paris, BnF, nouv. acq. fr. 12665. J. Hellevin a lui-même copié cet exemplaire français de l'Horloge de sapience de Henri Suso, à la fin duquel il a également signé «J. Hellevin ${ }^{31} »$. Ce manuscrit atteste que la scripta de J. Hellevin est également picarde ${ }^{32}$. Son exemplaire des Grandes Chroniques, le

${ }^{29}$ Voir les filigranes n ${ }^{\circ} 82251-82253$ du répertoire Piccard-Online.

30 Le français était assurément la langue privilégiée dans le monde littéraire bourguignon, et le patronage d'auteurs francophones par les ducs de Bourgogne exerça quelque rôle social dans l'identification du français comme langue de culture des Pays-Bas (Tania Van Hemelryck, «Qu'est-ce que la littérature... française à la cour des ducs de Bourgogne? », dans La Cour de Bourgogne et l'Europe. Le rayonnement et les limites d'un modèle culturel. Actes du colloque international tenu à Paris les 9, 10 et 11 octobre 2007, dir. Werner Paravicini, Ostfildern, Thorbecke, 2013, p. 351-359). L'emploi du français ne fut toutefois pas imposé aux structures locales, ainsi que l'illustrent les nominations d'officiers dans le duché de Brabant (Charles A. J. Armstrong, « The language question in the Low Countries. The use of French and Dutch by the dukes of Burgundy and their administration », dans Id., England, France and Burgundy in the fifteenth century, Londres, Hambledon Press, 1983, p. 189-212). Sur l'usage administratif des langues dans le duché de Brabant avant la réunion des différentes provinces des Pays-Bas sous l'autorité des Valois de Bourgogne, voir Godfried Croenen, «Latin and the vernaculars in the charters of the Low Countries. The case of Brabant", dans The Dawn of the written vernacular in Western Europe, dir. Michèle Goyens et Werner Verbeke, Louvain, Leuven University Press, 2003, p. 107-125.

31 Colophons des manuscrits occidentaux des origines au XVI siècle, éd. Bénédictins du Bouveret, Fribourg, Éditions universitaires, 1973, t. 3, p. 8 [nं7462]. Issu de la collection Barrois passée à Ashburnham, ce manuscrit fut vendu chez Sotheby's en 1901 (Catalogue of the portion, op. cit., p. 112 [ $\mathrm{n}^{\circ} 299$ ]). Il passa ensuite en vente chez Jacques Rosenthal, puis fut acquis par la Bibliothèque nationale à la vente de la bibliothèque de Lucien Gougy en 1935.

${ }^{32}$ Voir la description donnée de ce volume, lors de son entrée dans les collections de la Bibliothèque nationale, par Philippe Lauer, « Nouvelles acquisitions latines et françaises du département des manuscrits de la Bibliothèque nationale pendant les 
manuscrit Paris, BnF, nouv. acq. fr. 6225, produit dans les Pays-Bas, n'avait donc pas quitté cette région quand ont été consignés dans ses marges des indices de sa réception.

En effet, le texte des Grandes Chroniques a été glosé dans les marges cet exemplaire ; la majorité du travail d'annotation se rapporte à la Flandre et au Brabant et à la lignée des princes qui se succèdent à la tête de ces provinces ${ }^{33}$. Ainsi rencontre-t-on des marques signalétiques se rapportant à la Flandre (comme «Jehan de Flandres, seigneur de Neelle tué par ceulx de Courtray ${ }^{34}$, «[le] roy de France envoie a Bruges pour la délivrance du conte [de Flandre] ${ }^{35} »$, ou « Lille assiegee par les Anglois ${ }^{36} »$ ) et au duché de Brabant («appareil du duc de Gheldres contre le duc de Brabant a cause de la conté de Luxembourg $^{37} »$, « mariages de Henry et Godeffroy de Brabant $\left.{ }^{38} »\right)$. En outre, à la fin du manuscrit, Hellevin replace cette chronique dans le contexte de sa bibliothèque personnelle, de toute évidence celle d'un

années 1932-1935 », Bibliothèque de l'École des chartes, vol. 96, 1935, p. 205245, cit. p. 234.

${ }^{33}$ Sur les quatorze notes significatives qui se rencontrent dans les marges de ce volume, treize sont de la même main. Parmi celles-ci, seules quatre ne se rapportent pas aux principautés des Pays-Bas.

34 Paris, BnF, nouv. acq. fr. 6225, fol. 87r, en regard du texte: «Sy y fu tué monseigneur Jehan de Flandres, autrement dit de Nelle, le conte de Flandres » (voir Les Grandes Chroniques de France, éd. Jules Viard, op. cit., t. 9, p. 47).

${ }^{35}$ Paris, BnF, nouv. acq. fr. 6225 , fol. 87r, en regard du texte : « et toutesfois ne fu point mis le conte hors de prison, dont il advint que le roy de France envoia messages sollempneus a Bruges » (voir Les Grandes Chroniques de France, éd. Jules Viard, op. cit., t. 9, p. 47).

${ }^{36}$ Paris, BnF, nouv. acq. fr. 6225 , fol. 145 r, en regard du texte : «Sy orent conseil les II contes et deliberation ensemble d'assegier Lille en Flandres » (voir Les Grandes Chroniques de France, éd. Jules Viard, op. cit., t. 9, p. 176).

37 Paris, BnF, nouv. acq. fr. 6225, fol. 2r, en regard du texte: «Tantost le duc de Gherles, qui au cuer prist ceste chose, manda tous ses parens et amis partout, et assambla sy trés grant ost que che fu merveilles » (voir Les Grandes Chroniques de France, éd. Jules Viard, op. cit., t. 8, p. 132).

${ }^{38}$ Paris, BnF, nouv. acq. fr. 6225 , fol. $194 \mathrm{v}$, en regard du texte : « et en che temps environ la saint Jehan Baptiste, le XXI ${ }^{\mathrm{e}}$ jour de juing, Henry et Godefroy, fils du duc de Breubant, furent espousés au Louvre a Paris » (voir Les Grandes Chroniques de France, éd. Jules Viard, op. cit., t. 9, p. 297). 
érudit passionné par l'histoire des Pays-Bas dans la seconde moitié du $\mathrm{XV}^{\mathrm{e}}$ siècle :

Monstrelet commenche sa chronique a Charles $\mathrm{VI}^{\mathrm{e}}$, roy de France, auquel ceste chronique fine. Ceste chronique commenche depuis l'an mil II $^{\mathrm{c}} \mathrm{IIII}^{\mathrm{xx}}$ VI et fine l'an mil III $^{\mathrm{c}}$ $\mathrm{IIII}^{\mathrm{xx}}$. Ma chronique de Liege est anterieure a ceste plus de [réserve] ans ${ }^{39}$.

Le chroniqueur Enguerrand de Monstrelet, au service de Philippe le Bon $^{40}$, et une Chronique de Liège, vraisemblablement un exemplaire de l'ouvrage de Jean d'Outremeuse s'interrompant en $1340^{41}$, servent ici à situer la chronologie du propos des Grandes Chroniques. Voilà donc trois ouvrages historiographiques mis au service des intérêts locaux d'un érudit, peut-être historien lui-même, travaillant aux marges du royaume.

D'autres manuscrits peuvent témoigner de réceptions «locales» des manuscrits des Grandes Chroniques, notamment dans le contexte des Pays-Bas bourguignons, perméables à la littérature française ${ }^{42}$. Ainsi le manuscrit Paris, BnF, fr. 4937-4938 a-t-il été annoté de façon

${ }^{39}$ Paris, BnF, nouv. acq. fr. 6225, fol. 423r. La réserve est le fait de l'annotateur.

40 La Chronique d'Enguerran de Monstrelet en deux livres, avec pièces justificatives. 1400-1444, éd. Louis Douët-d'Arcq, Paris, Renouard, 1857-1862, 6 vol.

${ }^{41}$ Jean des Preis dit d'Outremeuse, Ly Myreur des histors, éd. Adolphe Borgnet et Stanislas Bormans, Bruxelles, Hayez, 1865-1887, 7 vol. Jean d'Outremeuse a probablement rédigé un quatrième livre menant le propos du Myreur jusqu'aux événements de 1399; la diffusion médiévale de ce dernier aurait été quasi nulle, aucun exemplaire n'a survécu : voir Pierre Courroux, L'Écriture de l'histoire dans les chroniques françaises (XII $-X V^{e}$ siècle), Paris, Classiques Garnier, 2016, p. 316318.

42 Les ducs de Bourgogne possédaient de nombreux manuscrits des Grandes Chroniques, et des mécanismes de percolation dans la diffusion des ouvrages, liés au désir et à la rivalité mimétiques (ce que la sociologie désigne par l'expression trickle-down effect), ont pu être observés dans les Pays-Bas méridionaux (Céline Van Hoorebeeck, «La clientèle des miniaturistes. Des manuscrits enluminés pour quels publics ? », dans Miniatures flamandes. 1404-1482, dir. Bernard Bousmanne et al., Paris/Bruxelles, BnF/Bibliothèque royale de Belgique, 2011, p. 81-88, cit. p. 85). 
très précise par un lecteur attentif aux questions relatives à la Flandre, au Hainaut et au Vermandois ${ }^{43}$. Dans un manuscrit conservé à la Bibliothèque royale de Bruxelles sous la cote 14910-14912 $2^{44}$, à l'intérieur duquel des extraits des Grandes Chroniques servent de continuation aux Chroniques de Flandres, un annotateur a souligné tout ce qui concernait les villes de Gand et de Bruges, et plus généralement la Flandre, le Hainaut et le Brabant.

À ces attestations de réceptions «locales » de manuscrits modestes des Grandes Chroniques peut être assimilée celle, exceptionnelle, d'un exemplaire luxueux: le manuscrit Paris, BnF, fr. 2615. Si l'on ignore tout de l'itinéraire et des anciens possesseurs de ce manuscrit, on peut toutefois supposer que sa réception a eu lieu en Artois : ainsi, à chaque fois que le texte mentionne les comtes d'Artois, une croix a été placée en marge par un annotateur ${ }^{45}$.

\section{Utilisation professionnelle}

Dans l'espace francilien plus spécifiquement, la réception des Grandes Chroniques est attestée dans le contexte professionnel et institutionnel des offices de l'État royal; le manuscrit de la

\footnotetext{
43 Ancien manuscrit Colbert 2829-2830 et Regius 9615(7.7)-9615(8.8). En 1675, Étienne Baluze acquit, pour la bibliothèque de Colbert dont il avait la garde, ce manuscrit pour la somme de six livres, avec le reste de la collection de l'académicien Jean Ballesdens (1595-1675). Voir La Bibliothèque d'un académicien au XVII siècle. Inventaire et prisée des livres rares et des manuscrits de J. Ballesdens, suivis de son testament, éd. Léon Brièle, Paris, Imprimerie nationale, 1885, p. 12 [n $\left.{ }^{\circ} 542\right]$.

${ }^{44}$ Ce manuscrit est mentionné dans l'inventaire de la librairie du duc de Bourgogne Philippe le Bon dressé à Bruges vers 1467-1469 et dans celui de la librairie de Bruxelles en 1487: Corpus catalogorum Belgii. The medieval booklists of the Southern Low Countries, t. 5 : Dukes of Burgundy, dir. Thomas Falmagne et Baudouin Van den Abeele, Louvain/Paris/Bristol, Peeters, 2016, p. 248 et 334 $\left[\mathrm{n}^{\circ} 5.671\right.$ et 8.300].

${ }^{45} \mathrm{Ce}$ manuscrit apparaît pour la première fois dans la bibliothèque de Colbert, sous le $\mathrm{n}^{\circ} 350$.
} 
Bibliothèque nationale coté fr. 23143 en témoigne ${ }^{46}$. Constitué de plusieurs types de papier permettant de situer sa production autour de $1415^{47}$, ce codex opère dans les récits des règnes transcrits un choix plus restreint que le manuscrit de J. Hellevin. Le texte s'y limite en effet au récit des événements survenus durant les règnes des trois premiers Valois, qui s'échelonnent de 1328 à 1381.

À la fin du manuscrit figurent deux signatures : «J. de Longueil » et «P. Autissiodorensis episcopus ${ }^{48} »$. La première, grattée, est celle de Jean de Longueil, premier président du Parlement de Paris à l'époque anglo-bourguignonne ${ }^{49}$. La seconde, laissée par un évêque d'Auxerre dont le prénom débute par un $P$, ne peut être que celle du fils de Jean de Longueil, Pierre de Longueil. Outre ses fonctions au Parlement de Paris, Pierre fut évêque d'Auxerre entre 1449 et 1473. Le modeste manuscrit des Longueil permet donc d'enrichir la connaissance de la diffusion des manuscrits des Grandes Chroniques. Mais sans doute est-ce dans ce qu'il peut apporter à l'étude de la réception que réside son plus grand intérêt.

Les marges du manuscrit Paris, BnF, fr. 23143 ont été abondamment annotées, en français mais également en latin. Deux mains au moins se distinguent dans la rédaction des notes : une en latin, et une, voire davantage, en français. La stylisation des seings relevés à la fin du volume ne permet guère d'attribuer avec certitude l'une ou l'autre de ces mains aux Longueil eux-mêmes.

${ }^{46}$ Papier ; XV ${ }^{\mathrm{e}}$ siècle ; 275 x $200 \mathrm{~mm}$; 233 feuillets (sénions et octonions) ; 2 col. ; 31 1. Ce manuscrit constituait le $\mathrm{n}^{\circ} 430 \mathrm{du}$ fonds de la Sorbonne à la Bibliothèque nationale.

${ }^{47}$ Aux côtés de motifs très courants comme un léopard, une tête de bœuf, une ancre et un $Y$, un motif de cloche se distingue, qui peut être rapproché du Briquet 4081 (Troyes, 1415).

${ }^{48}$ Paris, BnF, fr. 23143, fol. 233v.

${ }^{49}$ À son propos, voir les renvois fournis par l'index dans l'ouvrage de Françoise Autrand, Naissance d'un grand corps de l'État. Les gens du Parlement de Paris. 1345-1454, Paris, Publications de la Sorbonne, 1981. 
Toujours est-il que l'on a, dans cet exemplaire, agrémenté le texte des Grandes Chroniques de véritables renvois bibliographiques. Lorsque les Grandes Chroniques évoquent l'assemblée du clergé ayant pris place à Vincennes en $1329^{50}$, une note marginale, partiellement rognée, ajoute qu'il est possible d'en apprendre davantage à ce sujet : «[...] de ce qui fut fait $[\ldots]$ en ce conseil par [...] docteur Pierre de Congniere $[\ldots]$ fu fait un livre $[\ldots]$ par messire Pierre $[\ldots]$ docteur en loiz le $[\ldots]$ livre est en la [...] du college $[\ldots]$ n a Paris ${ }^{51} »$. Il est plus que probable qu'il soit fait ici référence au Libellus Petri Bertrandi, un ouvrage désigné du nom de son auteur, Pierre Bertrand, qui fut le fondateur du collège d'Autun ${ }^{52}$. Un inventaire, dressé en juillet 1462, de la bibliothèque de cette institution indique bien qu'un exemplaire en

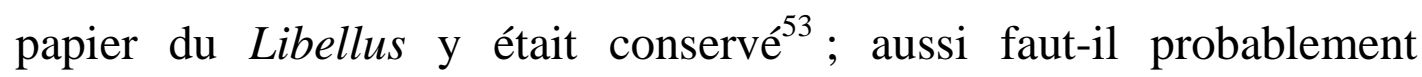
reconstituer la note en «college d'Autun ».

Quelques-unes parmi les nombreuses notes se rapportent directement, ou peuvent être rapportées, aux documents de la Chambre des comptes. Les richesses documentaires majeures de cette institution,

${ }^{50}$ Voir Les Grandes Chroniques de France, éd. Jules Viard, op. cit., t. 9, p. 113114.

${ }^{51}$ Paris, BnF, fr. 23143, fol. 15v.

52 À propos de cet ouvrage, voir Franz Olivier-Martin, L'Assemblée de Vincennes de 1329 et ses conséquences. Étude sur les conflits entre la juridiction lä̈que et la juridiction ecclésiastique au XIV siècle, Rennes, Bibliothèque universitaire, 1909, p. 52-64, qui étoffe le recensement des manuscrits conservés du Libellus établi par Jules Roy, «Conférence de Vincennes et conflits de juridiction (1329-1350)», dans Mélanges Renier. Recueil de travaux, Paris, Vieweg, 1887, p. 329-346, spéc. p. 345-346. - Il ne faut pas confondre le Libellus Petri Bertrandi avec le traité De origine jurisdictionum dont l'auteur est aussi Pierre Bertrand. À propos de ce dernier ouvrage, voir Franz Olivier-Martin, « Note sur le De origine jurisdictionum attribué à Pierre Bertrand », dans Mélanges Fitting. LXXV ${ }^{e}$ anniversaire de Hermann Fitting, Montpellier, Société anonyme de l'Imprimerie générale du Midi, 1908, t. 2 , p. 104-119; Franz Olivier-Martin, L'Assemblée de Vincennes, op. cit, p. 64-68; et en dernier lieu Carlos Larrainzar González, «El opúsculo De origine iurisdictionum del cardenal Pierre Bertrand », Initium. Revista catalana d'historia del dret, vol. 16, 2011, p. 131-172.

53 Alfred Franklin, Les Anciennes Bibliothèques de Paris. Églises, monastères, collèges, etc., Paris, Imprimerie impériale, 1867-1873, t. 2, p. 72. 
les mémoriaux partis en fumée en 1737 , ont notamment été mis à contribution. Désignés pour les premiers d'entre eux par des mots du Pater noster ou des signes, pour les suivants par des lettres de l'alphabet, ces registres consignaient les actes et traités émis par les rois de France ${ }^{54}$. Les Grandes Chroniques évoquent ou reproduisent in extenso un certain nombre de traités de paix et d'actes royaux. Ces insertions prennent une ampleur telle dans le récit des règnes de Jean II et surtout de Charles V que l'on a pu faire remarquer que, sur les 380 pages qu'occupe l'édition du récit du règne de ce dernier roi, 200 reproduisent des traités et des documents diplomatiques ${ }^{55}$. Un lecteur du manuscrit Paris, BnF, fr. 23143 opéra donc un rapprochement entre la partie des Grandes Chroniques attribuée à Pierre d'Orgemont et les mémoriaux de la Chambre des comptes.

Constatant que le scribe du manuscrit fr. 23143 s'était contenté de transcrire l'année de l'hommage rendu à Charles $\mathrm{V}$ par Jean de Montfort au titre du duché de Bretagne (1366) et avait ignoré le jour et le mois où eut lieu cet événement ${ }^{56}$, l'annotateur fut contraint de chercher lui-même ces informations qu'il consigna dans la marge : «le XIII ${ }^{\mathrm{e}}$ jour de decemb[...], comme porte le regi[...] de la chambre des comp[...], et illec sera veu tout la maniere des dits homm[...], ou livre signé $\mathrm{D}$, ou fueillet $\mathrm{CIX}^{57} »$.

\footnotetext{
54 Charles-Victor Langlois, «Registres perdus des archives de la Chambre des comptes de Paris », dans Notices et extraits des manuscrits de la Bibliothèque nationale et autres bibliothèques, t. 40, 1916, p. 33-399.

${ }^{55}$ Raymond Cazelles, Société politique, noblesse et couronne sous Jean le Bon et Charles V, Genève, Droz, 1982, p. 526. Sans entrer dans les mêmes considérations chiffrées, Roland Delachenal avait déjà souligné cette tendance: Les Grandes Chroniques de France, éd. Roland Delachenal, op. cit., t. 3, p. XX-XXI.

${ }^{56}$ Voir Les Grandes Chroniques de France, éd. Roland Delachenal, op. cit., t. 2, p. 24-25.

${ }^{57}$ Paris, BnF, fr. 23143, fol. 159r. Comme bien d'autres, cette note est rognée.
} 
Les Grandes Chroniques mentionnent également la décision de Charles V relative à la majorité du roi $(1374-1375)^{58}$, une décision qui allait au début du siècle suivant être invalidée par un acte de Charles VI. En marge de queue du manuscrit Paris, BnF, fr. 23143, on souligne que « chascun en fait a sa guise » avant d'expliquer le contenu de l'ordonnance du 26 décembre 1407 qui proclame l'instantanéité de la succession et le couronnement immédiat du successeur d'un roi défunt. « Laquelle loy ou constitution, conclut l'annotateur, est escripte ou livre septiesme des memoriaulx de la Chambre des comptes, signé $\mathrm{G}^{59} »$. Le simple renvoi bibliographique peut donc se muer, à l'occasion, en un petit appendice historique ${ }^{60}$.

Au-delà des renvois documentaires, des annotations laissées dans le manuscrit Paris, BnF, fr. 23143 témoignent également de la valeur accordée au texte et à l'origine des Grandes Chroniques de France.

${ }^{58}$ Ordonnances des rois de France de la troisième race [...], éd. Denis-François Secousse, Paris, Imprimerie royale, 1741, t. 6, p. 26-32. Voir Les Grandes Chroniques de France, éd. Roland Delachenal, op. cit., t. 2, p. 177-178.

59 Paris, BnF, fr. 23143, fol. 197v-198r. Voir le texte de cette décision de Charles VI dans les Ordonnances des rois de France de la troisième race [...], éd. Denis-François Secousse, Paris, Imprimerie royale, 1755, t. 9, p. 267-269. Elle a été également éditée à partir de l'ancien mémorial $\mathrm{G}$ dans le Recueil général des titres concernant les fonctions, rangs, dignitez, séances et privilèges des charges des présidens, trésoriers de France, généraux de finances et grands voyers des généralitez du royaume, éd. Simon Fournival, Paris, Chouqueux, 1655, p. 814-817. ${ }^{60}$ Un autre exemple de ce type se rencontre en regard de la mention du mariage conclu entre le futur Jean II et Bonne de Luxembourg (voir Les Grandes Chroniques de France, éd. Jules Viard, op. cit., t. 9, p. 132). Un annotateur a ajouté dans la marge, cette fois en latin, de nombreux éléments relatifs à cet événement que l'on pourra vérifier "per litteras regias in thesoro cartarum et privilegiorum regis existentes »(Paris, BnF, fr. 23143, fol. 21r). Contrairement aux indications relatives aux mémoriaux, rendues obsolètes par l'incendie de 1737 , ce renvoi est encore valide aujourd'hui, puisque le document concerné, daté du 17 février 1331 (a. st.), est conservé : Paris, AN, J 370, $\mathrm{n}^{\circ} 1$ (référence indiquée par Raymond Cazelles, Société politique, noblesse et couronne, op. cit., p. 457, n. 16). Le texte en a été édité, à partir de l'ancien mémorial $\mathrm{B}$ de la Chambre des comptes, par Nicolas Brussel, Nouvel Examen de l'usage général des fiefs en France pendant le XI ${ }^{e}$, le $X_{I I}^{e}$, le XIII ${ }^{e}$ et le XIV siècle, pour servir à l'intelligence des plus anciens titres $d u$ domaine de la couronne, Paris, Jacques Nicolas le Clerc, 1739 [1727], t. 2, p. LXXXX-LXXXXII. 
Celles-ci sont très souvent elliptiques lorsqu'il s'agit de désigner les grands princes du royaume. Comment connaître l'identité exacte de ce «duc de Bretaigne [qui] espousa la seur au conte de Savoye»? L'annotateur peste en marge: «c'est grant fault[...] ceulz qui croniq[...] qu'ilz ne mette[...] les noms des pri[...] et princesses ${ }^{61} »$. Ici se devine un esprit soucieux de clarté, peut-être accoutumé aux homonymies intrafamiliales qui obscurcissent l'interprétation des actes anciens. Résolvant lui-même les énigmes naissant de l'imprécision des Grandes Chroniques, l'annotateur indique en marge du texte qui parle du «duc de Normandie»: «c'est assavoir Jehan, l'ainsné filz du $\operatorname{roy}^{62} »$.

Ailleurs, le manque d'informations n'est plus seulement déploré ; un esprit critique met en cause la bonne foi du chroniqueur relatant les mouvements de l'armée anglaise autour de Paris au mois d'août 1346. Philippe VI est informé que

le roy d'Engleterre s'estoit party de Poissi et qu'il avoit fait refaire le pont qui estoit rompu, laquelle rompure avoit esté faicte, si comme Dieu scet, afin que le roy d'Engleterre ne peust eschapper sans soy combatre contre le roy de France ${ }^{63}$.

Cet appel de Dieu à témoin n'était visiblement pas du goût de tous, et dans la marge on a écrit : «il semble que l'acteur n'osa escrire la verité ou ce qu'il en pensoit ».

Mais qui est cet «acteur» ainsi critiqué ? Connaît-on bien, au $\mathrm{XV}^{\mathrm{e}}$ siècle, l'auguste origine de la chronique contenue dans le manuscrit Paris, BnF, fr. 23143 ? Rien n'est moins sûr. À propos d'un passage

${ }^{61}$ Paris, BnF, fr. 23143, fol. 13r (note rognée); voir Les Grandes Chroniques de France, éd. Jules Viard, op. cit., t. 9, p. 107.

${ }_{62}$ Paris, BnF, fr. 23143, fol. 62r.

63 Paris, BnF, fr. 23143, fol. 61r; voir Les Grandes Chroniques de France, éd. Jules Viard, op. cit., t. 9, p. 276-277. 
qu'il lit dans le récit du règne de Philippe VI, où l'auteur parle de « nostre maison de Rueil » pour signaler qu'elle dépend de l'abbaye de Saint-Denis ${ }^{64}$, l'annotateur commente en marge : «par ce appert que cellui qui fit ceste cronique estoit religieux de Saint Denis ${ }^{65}$ ». N'était-il pas évident pour notre homme, visiblement clerc de la Chambre des comptes et qui par ailleurs paraît bien informé, que les Grandes Chroniques dont il annotait un exemplaire étaient souvent dites de Saint-Denis ? Peut-être cela n'avait-il guère d'importance pour lui. Professionnel d'une institution qui avait la garde de la mémoire législative du royaume, il parcourait son manuscrit à la recherche de détails qui lui permettraient de mieux comprendre les documents dont la préservation était sous sa responsabilité ${ }^{66}$. Cette entreprise ne lui réussit guère: son exemplaire des Grandes Chroniques était assez mauvais et il le corrigea au moins autant qu'il n'en apprit.

L'une de ces corrections autorise l'hypothèse d'un annotateur officiant à la Chambre des comptes. En marge du texte des «lectres de renonciacions » qu'envoya Édouard III à Charles V en $1360^{67}$, cet annotateur prit la parole : «ceste lettres est escrip[...] ou $\mathrm{XXX}^{\mathrm{e}}$ fueillet [...] traittiéz d'Anglet[...] que j'ay fait escri[...]. Si soit illec corrigee,

\footnotetext{
${ }^{64}$ Les Grandes Chroniques de France, éd. Jules Viard, op. cit., t. 9, p. 275. La suite de la phrase diffère du texte édité, puisqu'on lit dans le manuscrit : « [...] nostre maison de Rueil, laquelle Charles le Chaufve roy et emperiere donna a l'eglise de Saint Denis », plutôt que, dans l'édition : «[...] nostre maison de Rueil, laquelle Charles le Chauve roy et emperere donna à nostre eglise ».

${ }^{65}$ Paris, BnF, fr. 23143, fol. 61r.

${ }^{66}$ Sur la culture historique et l'activité historiographique des clercs de la Chambre des comptes aux $\mathrm{XV}^{\mathrm{e}}-\mathrm{XVI}^{\mathrm{e}}$ siècles, voir Kathleen Daly, " "Pour vraye congnoissance avoir". Historical culture and polemic in the French royal Chambre des comptes in Paris in the fifteenth century », Nottingham medieval studies, vol. 49, 2005, p. 142189 ; Elizabeth A. R. Brown et Sanford Zale, « Louis Le Blanc, Estienne Le Blanc, and the defense of Louis IX's crusades. 1498-1522», Traditio, vol. 55, 2000, p. 235-292.

${ }^{67}$ Voir Les Grandes Chroniques de France, éd. Roland Delachenal, op. cit., t. 2, p. 47-58.
} 
car cy a plusieurs faultes ${ }^{68} »$. Doué d'autorité pour les questions archivistiques, notre homme devait donc occuper quelque poste à responsabilité à la Chambre des comptes.

\section{Le $\mathrm{XVI}^{\mathrm{e}}$ siècle antiquaire}

Le dernier exemple dont il sera question ici date du XVI ${ }^{\mathrm{e}}$ siècle. Il témoigne ainsi de la réception des Grandes Chroniques dans le temps long, au-delà des découpages académiques du continuum de l'histoire. La «sortie de mode ${ }^{69}$ » des Grandes Chroniques est un phénomène observable - ou plutôt déductible du silence progressif des sources - à partir du milieu du XVI ${ }^{\mathrm{e}}$ siècle. Sa datation exacte, sa caractérisation et son explication passent par l'étude de l'accueil réservé aux Grandes Chroniques par ces érudits de la France du XVI ${ }^{\mathrm{e}}$ siècle, dont les riches bibliothèques sont loin d'avoir livré tous leurs secrets ${ }^{70}$.

Jean Le Féron (1504-v. 1570) est l'un de ces érudits. Parlementaire, historien, généalogiste et héraldiste, Le Féron possède au milieu du XVI siècle une bibliothèque riche de 600 à 700 volumes, imprimés et manuscrits. Y figure un exemplaire des «Grandes croniques de France en langue françoise, escripte à la main » en trois

\footnotetext{
${ }_{68}^{6}$ Paris, BnF, fr. 23143, fol. 163r. Note rognée.

69 J'emprunte cette expression à Pascale Bourgain, «Les frontières. Codicologie des manuscrits occidentaux », dans Frontiers in the Middle Ages. Proceedings of the Third European Congress of medieval studies (Jyväskylä, 10-14 June 2003), dir. Outi Merisalo et Päivi Pahta, Louvain-la-Neuve, FIDEM, 2006, p. 387-398, cit. p. 395.

${ }^{70}$ En effet, la compréhension de ce qu'il est advenu du patrimoine manuscrit médiéval après 1500, et ce à quoi il était employé, bénéficierait grandement d'une multiplication des études comme celle menée par Jérôme Delatour, Une Bibliothèque humaniste au temps des guerres de religion. Les livres de Claude Dupuy d'après l'inventaire dressé par le libraire Denis Duval (1595), Villeurbanne, ENSSIB, 1998. Pour une perspective d'histoire intellectuelle, voir Accès aux textes médiévaux de la fin du Moyen Âge au XVIII siècle. Actes de colloque, dir. Michèle Guéret-Laferté et Claudine Poulouin, Paris, Champion, 2012.
} 
volumes, prisé à vingt sous tournois ${ }^{71}$. Seul le deuxième de ces trois volumes, le manuscrit Paris, BnF, fr. 4956, avait jusqu'ici été repéré par Léopold Delisle $^{72}$. J'ai identifié le premier dans le manuscrit Vatican, BAV, Reg. lat. $921^{73}$, mais j'ignore ce qu'il est advenu du troisième volume des Grandes Chroniques de Le Féron.

Quel usage Jean Le Féron peut-il bien faire des Grandes Chroniques de France? Cet avocat au Parlement de Paris sous François I $^{\text {er }}$ et Henri II est passionné de généalogie et d'héraldique, à tel point d'ailleurs que ses contemporains déplorent son manque d'activité au Parlement. Antoine Loisel, un avocat d'une trentaine d'années son cadet, dit de lui «qu'il s'adonna plus à escrire des genealogies et armoiries qu'à son estat d'advocat ${ }^{74} »$.

Jean Le Féron ajoute à son exemplaire quantité d'annotations marginales qui confirment largement le jugement de Loisel. Elles attestent en effet son intérêt pour les questions généalogiques, dynastiques et héraldiques, mais également pour ce qui touche à l'histoire des officiers de l'Hôtel du roi. L'érudit dessine en outre, dans les marges de son exemplaire, des blasons à l'encre noire lorsqu'apparaissent dans le texte des noms de personnages dont il

\footnotetext{
${ }^{71}$ Roger Doucet, Les Bibliothèques parisiennes au XVI siècle, Paris, Picard, 1956, p. 129 [n $\left.{ }^{\circ} 264\right]$.

${ }^{72}$ Léopold Delisle, Le Cabinet des manuscrits de la Bibliothèque nationale, Paris, Imprimerie nationale, 1874, t. 2, p. 377.

${ }^{73}$ Papier ; XV siècle ; 280 x $200 \mathrm{~mm} ; 330+384$ feuillets (maj. quinions) ; 1 col. ; 30-36 1. Après la mort de Jean Le Féron, son exemplaire des Grandes Chroniques passa dans la bibliothèque des Petau. En 1650, la reine Christine de Suède acheta la majeure partie des codices médiévaux d'Alexandre Petau et partit pour l'Italie où ses collections (y compris le manuscrit Vatican, BAV, Reg. lat. 921) furent rachetées par le pape Alexandre VIII. Le reste des collections de Petau fut acquis par différents bibliophiles au nombre desquels se trouvait le cardinal Mazarin. À la mort de ce dernier, une grande opération d'échange pilotée par Colbert permit à la Bibliothèque du roi d'entrer en possession d'une partie des collections mazarines, dont le manuscrit Paris, BnF, fr. 4956.

74 Antoine Loisel, Pasquier ou Dialogue des avocats du Parlement de Paris, éd. André Dupin, Paris, Videcoq, 1844, p. 103-104.
} 
prétend connaître les armoiries. Ces blasons répondent à la grande œuvre de Jean Le Féron. Comme il l'admet lui-même vers 1560, l'avocat a

emploié [son] temps rejectant toute vaccation servile et mercenaire a rediger en douze vollumes l'histoire armorialle, contenant les escussons, blasons, noms, surnoms, qualitez et mémoire perpétuelle des Roys, princes, seigneurs, gentilzhommes et nobles de plusieurs royaulmes chrestiens et infidelles ${ }^{75}$.

Si l'Histoire armoriale n'est pas imprimée sous cette forme, Le Féron publie néanmoins, en 1555 , une série de petits armoriaux individuels consacrés aux officiers de l'Hôtel du roi de France: connétables, grands-maîtres, maréchaux, amiraux, etc. ${ }^{76}$, ainsi qu'aux prévôts de Paris et aux ducs du royaume. Comme l'indiquent les titres donnés aux différents opuscules, Le Féron projette dans l'obscurité des origines mérovingiennes de la royauté la fondation des offices attachés à la personne royale. Son Catalogue des [...] connestables commence dès lors au roy Clotaire, et celui des illustres mareschaulx au roy Clovis $^{77}$.

Les armoiries fabuleuses que Le Féron attribue aux officiers mérovingiens et carolingiens se retrouvent, tracées par ses soins, dans les marges de son exemplaire des Grandes Chroniques, dont les volumes identifiés conservent un récit qui s'étend des origines troyennes jusqu'à la mort de Henri $\mathrm{I}^{\text {er }}$ (1060). Sans intérêt pour

${ }^{75}$ Cité par Hector de La Ferrière-Percy, Le Journal de la comtesse de Sanzay. Intérieur d'un château normand au XVI siècle, Paris, A. Aubry, nouv. éd., 1859, p. 141-142.

${ }^{76}$ Tous furent imprimés par Michel de Vascosan.

77 Jean Le Féron, Catalogue des tresillustres ducz et connestables de France, depuis le roy Clotaire premier du nom, iusques à trespuissant, tresmagnanime \& tresvictorieux roy de France, Henry deuxieme, Paris, Michel de Vascosan, 1555 ; Id., Catalogue des illustres mareschaulx de France, depuis le roy Clovis deuxieme du nom, iusques a trespuissant, tresmagnanime \& tresvictorieux roy de France, Henry deuxieme, Paris, Michel de Vascosan, 1555. 
l'héraldique, les Grandes Chroniques de Le Féron lui servent donc à mettre au point les petites notices biographiques dont il agrémente ses armoriaux, avec les ouvrages d'autres auteurs qu'il cite explicitement, tels Vincent de Beauvais, Robert Gaguin ou Raffaello Maffei dit Volaterrane.

Les sources des blasonnements fabuleux sont, pour certaines, transparentes. Jean Le Féron procède de manière régressive pour les princes féodaux. Par exemple, il attribue à Thierry $\mathrm{I}^{\mathrm{er}}$, duc de HauteLotharingie (v. 965-v. 1026), les armoiries que la maison d'Alsace a par la suite données à la Lorraine : «d'or à la bande de gueules chargée de trois alérions d'argent ${ }^{78} »$. De toute évidence, Le Féron ignore, ou feint d'ignorer, que le Thierry de l'an mil est en réalité issu de la maison d'Ardenne qui n'est plus, au XVI ${ }^{\mathrm{e}}$ siècle, en possession du duché de Lorraine ${ }^{79}$.

D'autres blasons reproduits en marge du texte des Grandes Chroniques s'expliquent moins aisément. D'où vient cet écu «de sable à l'aigle d'or, couronné de mesme, membré \& becqué d'azur », que Le Féron attribue à Conrad, comte de Paris, contemporain de Charles le Simple, et qu'il range au nombre des «grands maistres de France $^{80} »$ ? ou ce blason « d'or à l'aigle de synople, à la bordure semee de France » de «Bouchard ou Bochard, duc de France, \& le premier

\footnotetext{
${ }^{78}$ Le Féron a tracé ce blason dans le manuscrit Paris, BnF, fr. 4956, fol. 155r, en regard d'un passage où Primat dresse l'inventaire des individus enterrés à Belin, auxquels il ajoute lui-même Thierry de Lorraine (voir Les Grandes Chroniques de France, éd. Jules Viard, op. cit., t. 3, p. 285).

${ }^{79}$ Voir Michel Parisse, « Généalogie de la maison d'Ardenne », Publications de la section historique de l'Institut grand-ducal de Luxembourg, vol. 95, 1981, p. 9-41, cit. p. 25. Sur la succession des dynasties d'origine carolingienne à la tête du duché de Haute-Lotharingie, puis de Lorraine, voir encore Michel Parisse, Noblesse et chevalerie en Lorraine médiévale. Les familles nobles $d u X I^{e}$ au XIII siècle, Nancy, Publications de l'université de Nancy II, 1982, p. 83-121.

${ }^{80}$ Jean Le Féron, Catalogue des tresillustres grands-maistres de France [...], Paris, Michel de Vascosan, 1555, fol. 18r. Blason dessiné par Le Féron dans la marge du manuscrit Paris, BnF, fr. 4956, fol. 308v.
} 
portant le tiltre \& nom de Connestable de France ${ }^{81} »$ ? Une étude consacrée aux fondements intellectuels et livresques de l'activité érudite et antiquaire de Jean Le Féron apporterait certainement à ces questions d'intéressants éléments de réponse.

Certes, trois attestations d'utilisation particulière des Grandes Chroniques ne peuvent, d'une façon qui soit à la fois fidèle et exhaustive, représenter l'hétérogénéité des contextes de réception de cette œuvre. Toutefois, ces exemples évoquent la diversité des interprétations et dessinent, comme en pointillés, le périmètre de ce que peuvent être les différents contextes de réception, de lecture, de rapports au manuscrit et aux Grandes Chroniques. Entre disparités géographiques, usages institutionnels et réappropriations intellectuelles d'érudits ou d'amateurs, la question que l'étude de la réception des Grandes Chroniques de France pose en filigrane est celle de leur spécificité et de leur utilité aux yeux des médiévaux. Il s'agit de retrouver, dans le panorama littéraire tel qu'il était compris et vécu à la fin du Moyen Âge la juste place d'une œuvre à succès.

\footnotetext{
${ }^{81}$ Jean Le Féron, Catalogue des tresillustres ducz et connestables de France [...], Paris, Michel de Vascosan, 1555, fol. 14v. Ce blason est dessiné dans la marge du manuscrit Paris, BnF, fr. 4956, fol. 54r, en regard de la mention dans le texte de «Bulcart de Corse » (voir Les Grandes Chroniques de France, éd. Jules Viard, op. cit., t. 3, p. 107).
} 